This item was submitted to Loughborough's Research Repository by the author.

Items in Figshare are protected by copyright, with all rights reserved, unless otherwise indicated.

\title{
An Enhanced Feedback Scheme for Extended Orthogonal Space-Frequency Block Coded MISO-OFDM Systems
}

PLEASE CITE THE PUBLISHED VERSION

PUBLISHER

(c) IEEE

VERSION

VoR (Version of Record)

LICENCE

CC BY-NC-ND 4.0

\section{REPOSITORY RECORD}

Eltayeb, Nasr E.M., Shakiru K. Kassim, and Jonathon Chambers. 2019. "An Enhanced Feedback Scheme for Extended Orthogonal Space-frequency Block Coded MISO-OFDM Systems". figshare.

https://hdl.handle.net/2134/5384. 
This item was submitted to Loughborough's Institutional Repository (https://dspace.lboro.ac.uk/) by the author and is made available under the following Creative Commons Licence conditions.

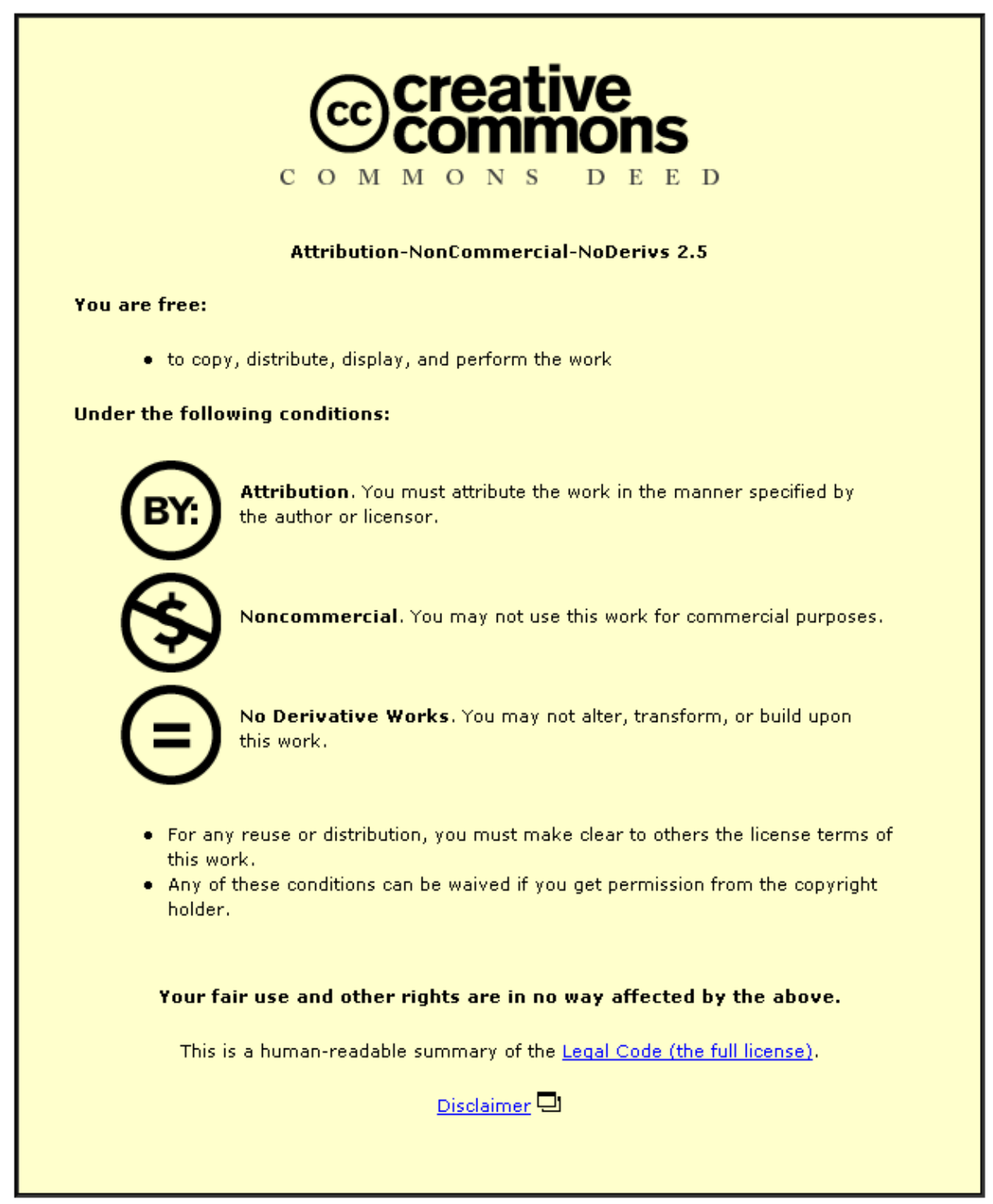

For the full text of this licence, please go to: http://creativecommons.org/licenses/by-nc-nd/2.5/ 


\title{
AN ENHANCED FEEDBACK SCHEME FOR EXTENDED ORTHOGONAL SPACE-FREQUENCY BLOCK CODED MISO-OFDM SYSTEMS
}

\author{
N. M. Eltayeb, S. K. Kassim, and J. A. Chambers \\ Advanced Signal Processing Group, Department of Electronic \& Electrical Engineering, \\ Loughborough University, Leicestershire, LE11 3TU, UK. \\ Email: \{n.e.eltayeb, s.k.kassim, j.a.chambers\}@lboro.ac.uk
}

\begin{abstract}
A simple extended orthogonal space-frequency coded multiple input single output (MISO) orthogonal frequency division multiplexing (OFDM) transmitter diversity technique for wireless communications over frequency selective fading channels is presented. The proposed technique utilizes OFDM to transform frequency selective fading channels into multiple flat fading sub-channels on which space-frequency coding is applied. A four-branch transmitter diversity system is implemented without bandwidth expansion and with only one receive antenna. The associated simulations verify that the four-branch transmitter diversity scheme achieves a significant improvement in average bit-error rate (BER) performance. The proposed scheme also outperforms the previously reported scheme due to $\mathrm{Yu}$ et. al. with only single phase feedback, and that improvement is retained with quantized feedback. Since the angle feedback is on a per tone basis, the feedback information would be too large for any practical OFDM system. However, we adopt a method which exploits the correlation among the feedback terms for the subcarriers, i.e. a group based quantization technique to reduce the feedback overhead significantly, rendering this scheme attractive to broadband wireless access systems.
\end{abstract}

\section{INTRODUCTION}

Spatial diversity combined with OFDM is an effective technique for mitigating detrimental effects in broadband wireless multipath fading channels. A key element of OFDM is the utilization of a large number of independent harmonically spaced sub-carriers which are formed in a computationally efficient manner with the fast Fourier transform (FFT). The use of multiple antennas for diversity can enhance channel capacity, making MIMO a very attractive practical system. Conventionally, multiple antenna systems have been employed at the receiver side. This increase of receiver hardware can be a major drawback, particularly for portable receivers where physical size and cost are restricted. Transmit diversity has received increased interest, typically for the downlink, as it can achieve diversity gain without increasing the size and complexity of the portable receiver.
Research on adapting several closed-loop methods for STBCs has been proposed to attain full code rate and full diversity. In [1], [2], [3] and the phases of the signals transmitted from certain antennas are rotated in a prescribed way in order to make the code orthogonal based upon the information fed back from the receiver, thereby increasing the diversity gain. Consequently, these techniques are often only effective over slowly-varying flat fading channels.

Space-time coded OFDM (ST-OFDM) systems [4] [5], have been proposed for frequency selective fading channels. In [5], it was shown that OFDM modulation with cyclic prefix can be used to transform frequency selective fading channels into multiple flat fading channels, so that orthogonal space-time transmitter diversity can be applied, even for channels with large delay spread. Another approach for transmission over MISO channels using OFDM is to replace the "time" dimension with the "frequency" dimension. The result is called space-frequency coded OFDM (SF-OFDM) transmitter diversity, which has also been suggested in [4].

In this paper, we extend this work to apply spacefrequency block coding (SFBC) with a $4 \times 1$ transmission matrix in [3], [2] and [1] to OFDM and compare the performance of the three systems in the presence of feedback. In particular, we develop a new scheme to maximize the signal to noise ratio (SNR) improvement as compared to previously reported work in [2]. For reference, quasi-orthogonal spacefrequency coded OFDM (QOSFBC-OFDM) has also been considered [1]. Simulation results show that the average symbol error rate performance of the proposed scheme outperforms previous schemes even when the scheme is quantized and when the fading channel is frequency-selective.

The organization of this paper is as follows. In Section II we describe the MIMO-OFDM system model and assumptions. In Section III, we introduce a general class of extended-orthogonal space-frequency block codes (EOSFBCs) (open-loop system) for frequency-selective fading channels. This class of EOSFBCs will then be used as an underlying structure to design full-diversity closed-loop space-frequency codes in Section IV. Simulation results are presented in Section V and finally some concluding remarks are provided in Section VI. 


\section{SYSTEM MODEL AND ASSUMPTIONS}

Figure 1 shows the baseband representation of the proposed closed-loop SF-OFDM transmitter diversity system. At the transmitter, let $X(m)$ denote the input data symbols with symbol duration $T_{s}$ first fed into a serial-to-parallel (S/P) converter with $m$ the symbol index. The data stream is then mapped across space and frequency by a space-frequency encoder in a QPSK constellation which then modulates the corresponding subcarrier. The resulting data vector is given by $\mathbf{X}(n)=\left[X_{0}(n) X_{1}(n) \ldots X_{N-1}(n)\right]^{T}$, where $X_{k}(n), k=$ $0,1, \ldots, N-1$ denotes the data symbols to be transmitted through a frequency selective fading channel of order $L$ on the $k$ th subcarrier during the block instant $n$. The notation $(.)^{T}$, represents the transpose operator. The modulated data symbols are then transformed by the inverse fast Fourier transform (IFFT) into an OFDM symbol. To avoid interframe interference (IFI) due to channel spread, a

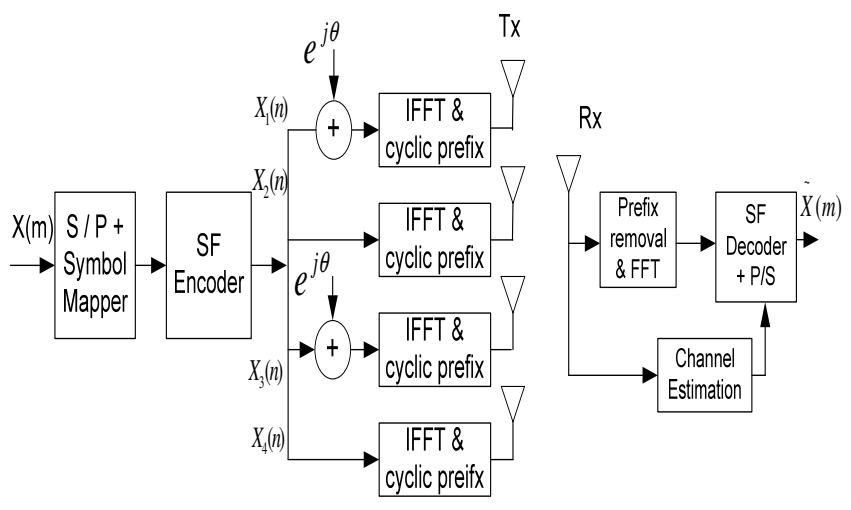

Fig. 1. Baseband representation of the proposed closed-loop space-frequency OFDM transmitter diversity system.

cyclic prefix with $c p$ symbols is inserted ahead of the block as a guard interval. The number of these cyclic prefix samples $c p$, should be greater than the channel order $L$, i.e., $c p>L$. We assume that the complex channel impulse response remains constant within a frame and changes only from one frame to another i.e., quasi-stationarity. At the receiver, these cyclic prefix samples are removed from the received signal. Then, a fast Fourier transform (FFT) is utilized to recover a block of received symbols. The demodulated signal vector is given by $\mathbf{R}(n)=\mathbf{\Lambda}(n) \mathbf{X}(n)+\mathbf{Z}(n)$, where $\boldsymbol{\Lambda}(n)$ is an $N \times N$ diagonal matrix whose diagonal elements are the FFT of the channel impulse response $h(n)$ and $\mathbf{Z}(n)$ is the channel noise vector.

\section{EXTENDED ORTHOGONAL SPACE-FREQUENCY CODED OFDM}

Space-frequency coding provides diversity gain by coding over spatial (antennas), and frequency dimensions. The proposed four-branch closed-loop EOSFBC-OFDM transmitter diversity system is an OFDM extension of closed-loop extended orthogonal transmitter diversity shown in [3].

The data symbol vector $\mathbf{X}(n)$ is coded into four vectors $\mathbf{X}_{1}(n), \mathbf{X}_{2}(n), \mathbf{X}_{3}(n)$, and $\mathbf{X}_{4}(n)$. by space-frequency encoder block as

$$
\begin{aligned}
& \mathbf{X}_{1}(n)=\mathbf{X}_{2}(n)=\left[X_{0}(n),-X_{1}^{*}(n), \ldots,-X_{N-1}^{*}(n)\right]^{T} \\
& \mathbf{X}_{3}(n)=\mathbf{X}_{4}(n)=\left[X_{1}(n), X_{0}^{*}(n), \ldots, X_{N-2}^{*}(n)\right]^{T}(1)
\end{aligned}
$$

where $(\cdot)^{*}$ denotes complex conjugate. During the block instant $n, X_{1}(n), X_{2}(n), X_{3}(n)$ and $X_{4}(n)$ are transmitted simultaneously from the first $T x_{1}$, second $T x_{2}$, third $T x_{3}$ and fourth $T x_{4}$ base stations respectively.

The equivalent space-frequency block code transmission matrix [2] is given by:

$$
\mathbf{C}=\left[\begin{array}{cccc}
X_{0}(n) & X_{0}(n) & X_{1}(n) & X_{1}(n) \\
-X_{1}^{*}(n) & -X_{1}^{*}(n) & X_{0}^{*}(n) & X_{0}^{*}(n)
\end{array}\right]
$$

where $X_{i}(n)$ is the OFDM symbol transmitted by the $i$ th transmit antenna at the $n$th frequency subcarrier.

The equivalent channel matrix corresponding to the code in (2) used over four transmitters is given by:

$$
\mathbf{H}(n)=\left[\begin{array}{cc}
\Lambda_{0}(n)+\Lambda_{1}(n) & \Lambda_{2}(n)+\Lambda_{3}(n) \\
\Lambda_{2}^{*}(n)+\Lambda_{3}^{*}(n) & -\Lambda_{0}^{*}(n)-\Lambda_{1}^{*}(n)
\end{array}\right]
$$

where $\Lambda_{i}, i=0,1,2,3$ are the channel coefficients modeled by independent zero-mean complex Gaussian random variables with 0.5 variance per dimension. The signal at the receiver is given by

$$
\begin{gathered}
{\left[\begin{array}{l}
R_{1}(n) \\
R_{2}^{*}(n)
\end{array}\right]=\mathbf{H}(n)\left[\begin{array}{l}
X_{0}(n) \\
X_{1}(n)
\end{array}\right]+\left[\begin{array}{l}
Z_{0}(n) \\
Z_{1}^{*}(n)
\end{array}\right]} \\
\mathbf{R}(n)=\mathbf{H}(n) \mathbf{X}(n)+\mathbf{Z}(n)
\end{gathered}
$$

Assuming the channel impulse responses are known or can be estimated accurately at the receiver, the space-frequency decoder constructs the decision estimate vector $\tilde{\mathbf{X}}(n)$ as

$$
\begin{gathered}
{\left[\begin{array}{c}
\tilde{X}_{0}(n) \\
\tilde{X}_{1}(n)
\end{array}\right]=\boldsymbol{\Delta}(n)\left[\begin{array}{c}
X_{0}(n) \\
X_{1}(n)
\end{array}\right]+\left[\begin{array}{c}
\tilde{Z}_{0}(n) \\
\tilde{Z}_{1}(n)
\end{array}\right]} \\
\tilde{\mathbf{X}}(n)=\boldsymbol{\Delta}(n) \mathbf{X}(n)+\tilde{\mathbf{Z}}(n)
\end{gathered}
$$

where $(\tilde{\cdot})$ denotes a quantity processed by $\mathbf{H}(n)^{H}$. It can be shown that for an orthogonal block code, all the offdiagonal terms of $\Delta=\mathbf{H}^{H}(n) \mathbf{H}(n)$ will be zero. It is thus reasonable to expect the proposed EOSF-OFDM transmit diversity system to achieve full diversity. However, the interference factor $\beta(n)$ as shown in equation (6) may reduce the performance of the scheme. In order to achieve maximum performance, i.e. full diversity and array gain, we propose a feedback scheme to modify the transmitted signals 
from certain antennas by rotating with an appropriate phase angle to ensure that the $\beta(n)$ term is maximized during the transmission period.

$$
\boldsymbol{\Delta}(n)=\left[\begin{array}{cc}
\alpha(n)+\beta(n) & 0 \\
0 & \alpha(n)+\beta(n)
\end{array}\right]
$$

where $\alpha(n)=\sum_{i=0}^{3}\left|\Lambda_{i}(n)\right|^{2}$ and the interference factor $\beta(n)=2 \operatorname{Re}\left\{\left(\Lambda_{0}(n) \Lambda_{1}^{*}(n)+\Lambda_{2}(n) \Lambda_{3}^{*}(n)\right)\right\}$. The operator $|\cdot|^{2}$ denotes the modulus squared of a complex number and $R e\{\cdot\}$ its real part.

\section{CLOSED-LOOP EOSF SCHEME FOR MISO-OFDM}

In [2], for a four antenna scheme, it was proposed in order to achieve full diversity, that the transmitted signals $s_{1}$ and $s_{3}$ are multiplied by $U_{1}(n)=(-1)^{i}$ and $U_{2}(n)=(-1)^{k}$, where $i, k=0$, or 1 , before they are transmitted from the first and third antennas, respectively; which means using 0 or $\pi$ as the rotation angle for the signals. Likewise in [7], the receiver computes the "interference factor" $\beta(n)$ and feeds back +1 if $\beta(n) \geq 0$ or -1 if $\beta(n)<0$. In our proposed scheme, the data signals transmitted from the first and third antennas are instead rotated by phase angles (phase shifted) $U_{1}(n)=e^{j \theta_{1}}$ and $U_{2}(n)=e^{j \theta_{2}}$ respectively while the other two are kept unchanged, where $\theta_{1}, \theta_{2} \in(-\pi, \pi]$. The phase element $e^{j \theta_{k}},(k=1,2)$ will introduce a delay which as in beamforming [8] will steer the energy in a certain direction which gives directivity and thereby chooses the direction that matches the channel. Therefore, the error performance is improved at the expense of increased feedback overhead. Beamforming techniques can be combined with space-frequency block coding to further improve the system performance. Those two technologies can be employed at the transmitter to provide transmit diversity and beamforming gain to increase the SNR of a wireless system. Examples of such systems are found in [9].

The phase rotation on the transmitted symbols is importantly effectively equivalent to rotating the phases of the corresponding channel coefficients.

The received signals $R_{1}(n)$ and $R_{2}(n)$, conjugated for convenience, at two independent time intervals are expressed as follows

$$
\begin{gathered}
{\left[\begin{array}{l}
R_{1} \\
R_{2}^{*}
\end{array}\right]=\left[\begin{array}{cc}
U_{1} \Lambda_{0}+\Lambda_{1} & U_{2} \Lambda_{2}+\Lambda_{3} \\
\left(U_{2} \Lambda_{2}\right)^{*}+\Lambda_{3}^{*} & -\left(U_{1} \Lambda_{0}+\Lambda_{1}\right)^{*}
\end{array}\right]\left[\begin{array}{c}
X_{0} \\
X_{1}
\end{array}\right]+\left[\begin{array}{c}
Z_{0} \\
Z_{1}^{*}
\end{array}\right]} \\
\mathbf{R}(n)=\mathbf{H}(n) \mathbf{X}(n)+\mathbf{Z}(n)
\end{gathered}
$$

where the implicit dependency on the block instant $n$ has been omitted for briefness. Assuming the complex channel gains between adjacent sub-carriers remain constant, the decision vector $\widetilde{\mathbf{X}}=\left[\tilde{X}_{0}(n), \tilde{X}_{1}(n)\right]^{T}$ with the receive vector $\mathbf{R}(n)=\left[R_{1}(n), R_{2}^{*}(2)\right]^{T}$ can be calculated as

$$
\begin{aligned}
\tilde{\mathbf{X}}(n) & =\mathbf{H}^{H}(n) \mathbf{R}(n)\left\{\left|U_{1}(n)\right|^{2}\left|\Lambda_{0}(n)\right|^{2}+\left|\Lambda_{1}(n)\right|^{2}\right. \\
& =+\left|U_{2}(n)\right|^{2}\left|\Lambda_{2}(n)\right|^{2}+\left|\Lambda_{3}(n)\right|^{2}+ \\
& =+2 \operatorname{Re}\left(U_{1}(n) \Lambda_{0}(n) \Lambda_{1}^{*}(n)\right) \\
& \left.=+2 \operatorname{Re}\left(U_{2}(n) \Lambda_{2}(n) \Lambda_{3}^{*}(n)\right)\right\} \mathbf{X}+\mathbf{H}^{H}(n) \mathbf{Z}(n)(8)
\end{aligned}
$$

Since $\left|U_{1}(n)\right|^{2}=\left|U_{2}(n)\right|^{2}=1$, then the channel gain, $g$ is $g=\sum_{i=0}^{3}\left|\Lambda_{i}(n)\right|^{2}+2 \operatorname{Re}\left\{\left(\Lambda_{0}(n) \Lambda_{1}^{*}(n) e^{j \theta_{1}}+\Lambda_{2}(n) \Lambda_{3}^{*}(n) e^{j \theta_{2}}\right)\right\}$

where $\theta_{k}, k=1,2$ are obtained by maximizing $\beta(n)$, and correspondingly the SNR, as follows:

$$
\begin{aligned}
& \theta_{1}=- \text { angle }\left(\Lambda_{0}(n) \Lambda_{1}^{*}(n)\right) \\
& \theta_{2}=- \text { angle }\left(\Lambda_{2}(n) \Lambda_{3}^{*}(n)\right)
\end{aligned}
$$

where angle $(\cdot)$ denotes the phase angles, in radians, for each complex element.

\section{IV-A. Common Phase-Rotation}

If $\theta_{1}=\theta_{2}$, a common phasor $e^{j \theta}$ can be used to rotate the transmitted symbols from the first and third antennas and the rotation angle is selected from a range between 0 and $2 \pi$. It is apparent that this does not change the transmitted power. Since the phase rotation on the transmitted symbols is effectively equivalent to rotating the phases of the corresponding channel coefficients, the new channel gain can be written as

$g=\sum_{i=0}^{3}\left|\Lambda_{i}(n)\right|^{2}+2 \operatorname{Re}\left\{\left(\Lambda_{0}(n) \Lambda_{1}^{*}(n)+\Lambda_{2}(n) \Lambda_{3}^{*}(n)\right) e^{j \theta}\right\}$

In this case, the transmitter needs to have knowledge of a single phase. Therefore, $\theta$ is determined as

$$
\theta=-\operatorname{angle}\left(\Lambda_{0}(n) \Lambda_{1}^{*}(n)+\Lambda_{2}(n) \Lambda_{3}^{*}(n)\right)
$$

\section{IV-B. Quantization}

Due to practical limitations, the number of feedback bits required from the receiver to the transmitter should be kept as small as possible. In the sequel, we provide a method to reduce the number of feedback bits while retaining satisfactory forward link performance. Feeding back the exact value of the phase angle with, for example, fixed or floating point resolution requires very large feedback overhead. In a practical application this may not be possible due to the very limited feedback bandwidth. Therefore, the phase angles should be quantized, and then these levels are fed back to the transmitter. Suppose, for each phase angle, if we have two bit feedback, then we can feedback only four phase level angles such that the phase angles are from the set of 
$\left\{\Theta_{1}, \Theta_{2}\right\} \in \Omega=[0, \pi / 2, \pi$ or $3 \pi / 2]$ then for the first antenna phase adjustment, the discrete feedback information corresponding to the phases may be selected according to

$$
\Theta_{1}=\arg \max _{\theta_{1} \in \Omega} \operatorname{Re}\left\{\left(\Lambda_{0}(n) \Lambda_{1}^{*}(n)\right) e^{j \theta_{1}}\right\}
$$

Similarly, for the third antenna phase adjustment, the phases may be selected according to

$$
\Theta_{2}=\arg \max _{\theta_{2} \in \Omega} \operatorname{Re}\left\{\left(\Lambda_{2}(n) \Lambda_{3}^{*}(n)\right) e^{j \theta_{2}}\right\}
$$

In this case the particular selection giving the largest values of (13) and (14) may be preferable, as it would provide the largest array gain and achieve full diversity advantage. This idea can also easily be applied to (12). We now make an observation that when the phase angle is not quantized and when both the first and third antenna signals are rotated using the phase angles, this scheme can be considered as a combined beamformer and SFBC [9], where the pairs of first and second antennas and third and fourth antennas are used as beamformers. Moreover, when the phase angles are quantized to only two levels ( 0 or $\pi$ ), this scheme is reduced to the scheme proposed in [7]. Hence, the proposed scheme is expected to perform between the scheme in [7] and combined beamformer and SFBC.

\section{SIMULATION RESULTS}

In this section, simulation results are provided to demonstrate the significant performance improvement achieved by the proposed EOSFBC coded OFDM system. First, we briefly describe the simulation parameters of the MISO-OFDM system.

An OFDM system with four transmit antennas and one receive antenna is used. The available channel bandwidth is divided into 64 tones. A cyclic prefix $c p$ larger than channel order is appended to each frame to combat inter-frame interference (IFI). In SF-Coding, QPSK constellations will be used. Frequency selective fading channels are used for simulation and average bit error rate (BER) will be used as the measure of performance. As highlighted previously, perfect channel state information was assumed to be available at the receiver and for SF-decoding the channel frequency responses of two adjacent sub-carriers were assumed to be identical. This technique can be extended straightforwardly to multiple receive antennas, but in this work, for simplicity, we consider only one receive antenna.

In order to compare the performance of our proposed closed-loop EOSF coded OFDM with the previously reported closed-loop EOSF coded OFDM schemes at the same spectral efficiency, the space-frequency (SF) QPSK coded OFDM scheme proposed in [2] is considered. For reference, we also provide a comparison of the proposed scheme with closed-loop QOSF [1]. Due to practical limitation, a quantized scheme scenario is also considered. We also

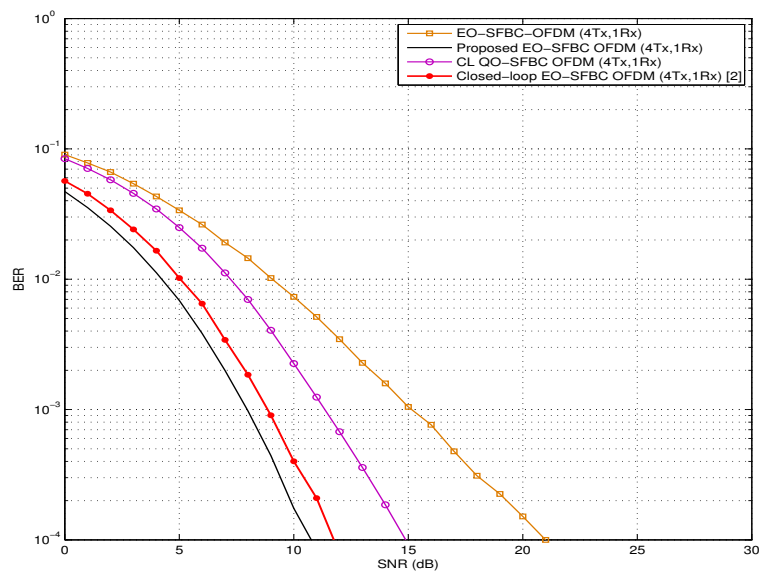

Fig. 2. Performance comparison of BER for different spacefrequency block codes; four transmit antennas, one receive antenna.

illustrate the performance /feedback-rate tradeoff and suggest possible options in easing this tradeoff.

Figure (2) shows the performance comparison of the previously reported SF coded OFDM versus proposed SF coded OFDM system. It is shown that, our proposed scheme outperforms the previous scheme [2] by more than $6 \mathrm{~dB}$ for open loop and $1 \mathrm{~dB}$ for closed loop at a bit error rate (BER) of $10^{-2}$ and about $7.5 \mathrm{~dB}$ and $1 \mathrm{~dB}$ of gain at a BER of $10^{-3}$ for open loop and closed loop respectively. Also shown in figure (2) is the performance curve of the QOSF coded OFDM which does not benefit from the array gain of the other scheme. The performance of the proposed scheme provides approximately $3 \mathrm{~dB}$ of gain at BER of $10^{-3}$ as compared to QOSF.

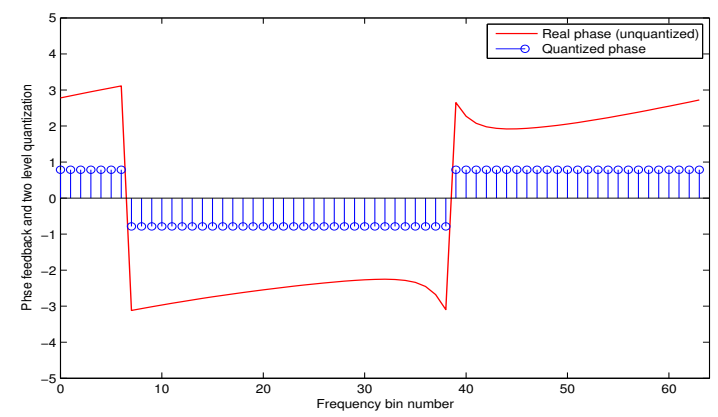

Fig. 3. The phase rotation angles.

The simulation result of a practical scenario of a quantization method is depicted in figure (4). In addition to this, the un-quantized (ideal phase) feedback for the proposed scheme is also depicted. From the figure, simulation results indicate 
that the performance of the quantized scheme is very close to the performance of the un-quantized scheme, and still significantly better than that of the previously reported closed loop scheme [2]. For phase rotation method, we quantized the required phase rotation angle into four levels $[0, \pi / 2, \pi$, $3 \pi / 2$ ] so that only two bits per subcarrier were required for feedback.

For frequency division duplex (FDD) based OFDM, however, we require at least $\mathrm{N}$ bits feedback for the phase rotation method. This imposes too much of an overhead constraint on the uplink channel to convey very large amount of feedback information. However, for OFDM based WLANs and WMANs (terminals are likely to remain fixed), the channel variations are small as compared to mobile channels in UMTS, hence phase angles can be fedback less frequently. Nevertheless, techniques that could potentially reduce the amount of feedback will yield significant benefits to the broadband wireless access systems. One possibility to reduce feedback overhead is to exploit the strong correlation in the feedback sequence among the subcarriers [6].

For an arbitrary frequency selective channel of length two, we computed the phase feedback required for a 64 point OFDM symbol and depicted in figure (3). A two level quantized phase $-\pi / 4$ and $\pi / 4$ is also shown in the same figure. We observe that the required phase feedback is a highly correlated waveform in frequency. We exploit this to perform significant reduction in feedback overheads.

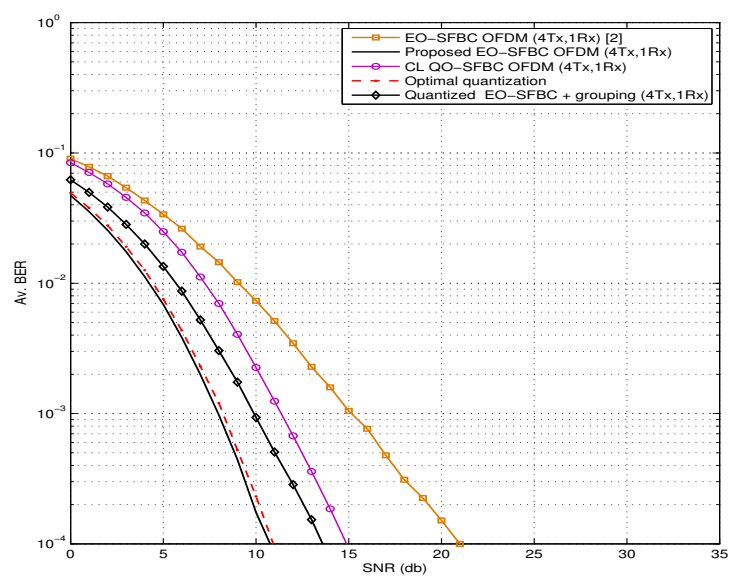

Fig. 4. Comparison of BER performance of the new scheme using quantized channel information with four transmit and one receive antennas, with per-tone quantization and grouping of feedback terms based quantization, with $\mathrm{N}=4$.

As a tradeoff, we assigned $\mathrm{L}$ number of consecutive subcarriers into a group and proposed to feedback one quantized phase angle for each group instead of feeding back the phase angle for each subcarrier. The phase angle required for each group is determined based on the majority of phase angles within that group. In this way, the 64 bits feedback required for one OFDM symbol is reduced to only $P=64 / L$ bits. Note that even with $P=4$, the result shown in figure (4) remains as an improvement over the original scheme [2]

\section{CONCLUSIONS}

In this paper, we described an extended orthogonal spacefrequency coded OFDM scheme for providing high datarate wireless communication over frequency selective fading channels. We presented simulation results for a QPSK spacefrequency coded OFDM system and compared its performance to the previously reported space-frequency coded OFDM scheme of [2]. We showed that the proposed scheme is capable of reliable transmission at relatively low SNRs making it a robust alternative technique, and suggested a scheme to reduce the feedback overhead to allow practical realization. Further exploitation of the multipath diversity is subject of on-going research.

\section{REFERENCES}

[1] C. Toker, S. Lambotharan, and J. A. Chambers, "ClosedLoop Quasi-Orthogonal STBCs and their Performance in Multipath Fading Environments and When Combined With Turbo Codes," IEEE Trans. Wireless Communications, Vol.3(6), pp. 1890-1896, Nov. 2004.

[2] Y. Yu, S. Keroueden, and J. Yuan, "Closed-Loop Extended Orthogonal Space-Time Block Codes for Three and Four Transmit Antennas," IEEE Signal Processing Letters, Vol.13, No.5, pp. 273-276, May 2006.

[3] N. M. Eltayeb, S. Lambotharan, and J. A. Chambers, "A Phase Feedback Based Extended Space-Time Block Code for Enhancement of Diversity," IEEE VTC Conference, IEEE 65th, pp.2296-2299, April 2007.

[4] D. Agrawal, V. Tarokh A. Naguib, and N. Seshadri , "SpaceTime Coded OFDM for High Data-Rate wireless Communication Over Wideband Channels," Vehicular Technology Conference, VTC 48th IEEE Vol 3, pp.2232-2236, May 1998.

[5] K. F. Lee, and D .B. Williams, "A Space-Time Coded transmitter diversity technique for frequency selective fading channels," in Proc. IEEE Sensor Array and Multichannel Signal Processing Workshop, Cambridge, MA, pp.149-152, March 2000.

[6] S. Lambotharan, and C. Toker, "Closed-loop space time block coding techniques for OFDM based broadband wireless access systems," IEEE Trans. Consumer Electronics, Vol.51, pp.765769, August 2005.

[7] J. Akhtar and D. Gesbert, "Extended Orthogonal Block Codes With Partial Feedback," IEEE Trans. Wireless Commun., Vol.3, no.6, pp.1959-1962, Nov. 2004.

[8] G. Jongren, M. Skoglund, and B. Ottersten, Combining beamforming with orthogonal space-time block coding, IEEE Trans. Inform. Theory, Vol. 48, pp. 611627, 2002.

[9] L. Hongwu, and L. Ji, "Space-Frequency OFDM System with Null-Steering Beamformer," ITS Trans. Inf. Theory, pp.341344, June 2006. 